ScIDice

\section{Non-Suturing Methods of Microvascular Anastomosis in Maxillofacial Reconstruction: A Literature Review}

Research Article

\author{
Ashutosh Deshpande ${ }^{1}$, Hemavathy OR ${ }^{2}$, M. P. Santhosh Kumar ${ }^{3 *}$
}

${ }^{1}$ Postgraduate Student, Department of Oral and Maxillofacial Surgery, Saveetha Dental College and Hospitals, Saveetha Institute of Medical and Technical Sciences (SIMATS), Saveetha University, Chennai 600077, Tamil Nadu, India.

${ }^{2}$ Professor, Department of Oral and Maxillofacial Surgery, Saveetha Dental College and Hospitals, Saveetha Institute of Medical and Technical Sciences (SIMATS), Saveetha University, Chennai 600077, Tamil Nadu, India.

${ }^{3}$ Professor, Department of Oral and Maxillofacial Surgery, Saveetha Dental College and Hospitals, Saveetha Institute of Medical and Technical Sciences (SIMATS), Saveetha University, Chennai 600077, Tamil Nadu, India.

Abstract

\begin{abstract}
New surgical ideas and techniques are introduced when surgical processes get more sophisticated, in order to make operations easier and reduce working time. Suturing procedures have long been the mainstay for microvascular anastomoses, however due to technological issues and labour intensity, sutureless microvascular anastomoses have advanced significantly. In this review, we discuss the trends of this era through the years, with a focal point at the greater current traits of laser-assisted vascular anastomoses, the coupler system, vascular closure staples and fibrin adhesives in maxillofacial reconstruction. The running principles of various methods of non-suturing methods of microvascular anastomosis in maxillofacial reconstruction along with their advantages and disadvantages are discussed in this review article.
\end{abstract}

Keywords: Anastomosis; Microsurgery; Microvascular; Review; Sutureless; Microvascular Reconstructive Surgery.

\section{Introduction}

On a large scale, suturing techniques predominate in both experimental and clinical contexts, with only a small amount of interest in alternative mechanical procedures. To improve the efficacy of anastomosis techniques, fine instruments such as a microscope, fine operating instruments, and fine suture materials are required. To improve surgical techniques from macroscopic to microscopic, knowledge and clinical practise with this precise operating equipment must be made aware. This review discusses the recently developed microvascular anastomosis techniques $[1,2]$.

Although sutured anastomosis has been shown to be effective, there are a number of drawbacks to the material utilised and the procedure itself. The suture material's downsides include the protrusion of prothrombotic suture material into the vessel lumen and myointimal hyperplasia caused by a foreign body reaction in the blood vessel wall. Furthermore, the entire procedure is time consuming and may result in considerable blood vessel damage, as the suture needle produces insult to the medial and intimal layers, resulting in vasospasm. In light of this, studies on various vascular anastomosis methods such as fibrin adhesives, couplers, staples, and lasers have been conducted since the $1900 \mathrm{~s}$, with promising results. The first vascular anastomoses utilising a neodymium: yttrium-aluminium-garnet laser were reported by Jain and Gorisch. Payr's concept of interlocking magnesium rings for vessel ring anastomosis began in 1904 [3]. Small pins on one side kept the vessel ends everted, and the pins were bent to secure the anastomosis after passing through both vessel walls and the holes in the matching ring. Landon invented a metal ring with five slightly everted teeth on one end and a smooth end in 1913. Kirsch proposed the microvascular anastomosis in 1992, based on the notion of flanged, extra-vascular, intimal approximation by stainless steel arcuate-ledges clips. Extravascular staples were used to hold the vessel ends together after 90 degrees of eversion.

Sutureless devices ensure good intima-to-intima contact, resulting in high patency rates, shorter operating times, and less need for

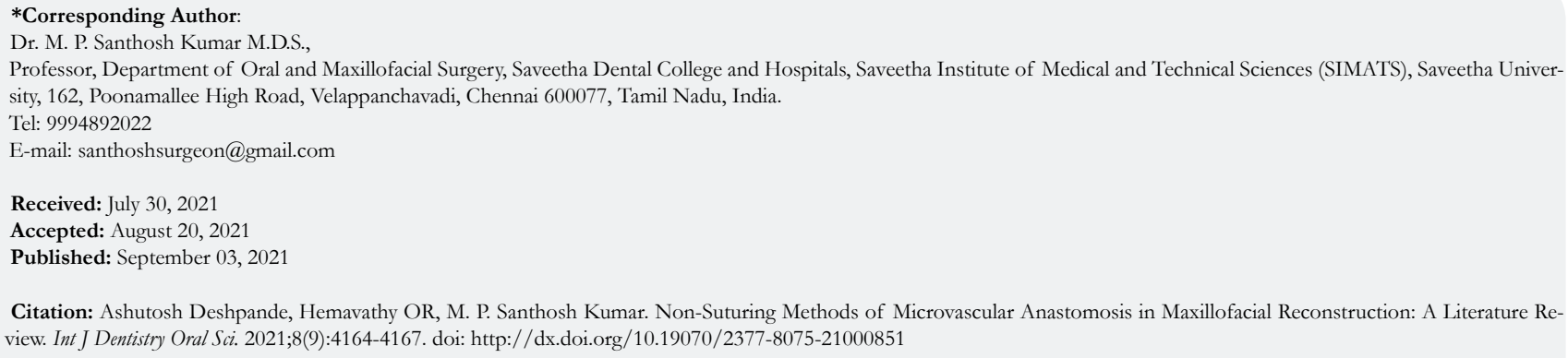

Copyright: M. P. Santhosh Kumar M.D.S. ${ }^{2}$ 2021. This is an open-access article distributed under the terms of the Creative Commons Attribution License, which permits unrestricted use, distribution and reproduction in any medium, provided the original author and source are credited. 
expertise and training [4]. This makes microsurgical techniques more accessible to surgeons with less experience. Certain technologies just outperform suturing materials in terms of efficacy and speed. The Coupler for venous anastomosis is one of the better examples. However, because of their complexity and burdensome instrumentation, they come with a slew of generic drawbacks. It demonstrates decreased vessel distensibility, is ineffective for considerable vessel size differences or end-to-end anastomoses, and results in greater vessel consumption into the device. This feature is not recommended for learning youngsters since it prevents attaining increased vessel diameter.

\section{Literature Search and Data Extraction}

The PubMed index [Medline database] was searched for research published till May 2020 those were limited to human subjects. Sutureless microvascular anastomosis, vascular closure staples, laser, tissue adhesives, and Coupler were the search terms. To support the data in the discussion and frame the introduction of the article, English-language clinical trials, review articles and meta -analysis research were gathered. For this review, the references were evaluated, and the relevant publications were included. The author's name, the type of study, the year, the type of patients, the anastomosis procedures employed were all extracted. To identify the reasonable and useful articles and to establish the art of the method, all parameters involved in the non-suturing microvascular anastomosis procedures were applied.

\section{Discussion}

Though the outcome of reconstruction is dependent on a number of parameters, preserving a patent microvascular anastomosis is critical. Less donor sites allow for better flap harvesting, in setting, and operating time, all of which lessen problems. The ischemic time of flap is measured from the time the flap is separated from the donor site until the vascular clamps are released after the anastomosis is completed. During this phase, vessels are prepared and the flap is partially in-set (devoid of blood supply). According to Murugan et al., as the flap ischemia time decreases, the likelihood of success increases significantly. The flap's survival is also influenced by injury to the deeper anatomical layers and the resulting vascular spasm. As a result, the anastomosis technique, which damages the deeper layer of the vessel during the procedure, drastically reduces blood flow following anastomosis.

Thomson et al., in 1995 concluded micro-suturing involves the endothelial layer, which eventually leads to thrombus development. These thrombi cause turbulence, which promotes thrombus development and full arterial obstruction by increasing the number of blood cell collisions with neighbouring areas of stasis. Intraluminal heparin irrigation is effective in preventing thrombus because it possesses endothelium binding capacity, inhibiting platelet aggregation, was postulated by Samuels and Webster in 1952. However, no effect on the macroscopic or microscopic findings was observed after irrigation of the lumen during surgery. The overall flap ischemia time is determined by the time spent preparing the vessels.

The flap ischaemic time is affected by a prolonged time of vessel preparation. Venous anastomosis is more challenging than the arterial anastomosis because the veins are low-pressure, thin-walled and are compromised readily by kinking, torsion, or external compression. According to Zhang et al., (2011), following anastomosis, veins are prone to spontaneous congestion, jeopardising the flap's venous return. They performed intra oral anastomosis for reconstructing midface defects and severe defects of alveolar ridge with short pedicled flaps. In flaps with shorter pedicles, they reported a faster flap harvesting time.

With the invention of newer technologies, usage of micro-staplers, couplers, and diode lasers are becoming popular due to easy application with minimal vessel preparation and minimal or noninvolvement of the endothelial layer [5].

\section{Fibrin Adhesives}

The fibrin glue mimics the final steps of the coagulation cascade to produce a physiologic fibrin clot. It was first used in microvascular anastomosis by Matras et al. and Pearl et al in 1977 [6]. Fibrin-reinforced anastomosis is a substitute to the other techniques of microvascular anastomosis. However, an easier anastomosis is achieved by using fewer sutures and sealing the inter suture gap with fibrin adhesive. In comparison with previous non-suturing approaches, fibrin glue enhanced suturing does not entirely eliminate endothelial involvement. The amount of insult to the endothelial layer is greatly reduced due to fewer sutures being placed [7]. The anastomotic time by this technique is almost equal to the time taken for anastomosing using conventional suturing despite placing fewer sutures, as the formulation of fibrin sealant requires additional time.

The main drawback of using fibrin glue is the risk of glue leakage into the vessel, that can also result in the formation of thrombus, which can then become an embolus [8]. Fibrin glue's sealant activity has a positive impact on preserving the anastomosis without any leakage from the site and stabilises the anastomosis, reducing vessel congestion[2]. Drake and Frost-Arner et al. contradicted this by demonstrating the use of lower doses of thrombin in conjunction with fibrinogen which did not promote thrombogenicity in epigastric free flap models [9].

\section{Vascular Couplers}

In 1962, Nakayama et al. developed a device consisting of two metal rings with 12 interlocking pins and corresponding holes, which they called a coupler [10]. The ends of the donor and recipient vessels were intended to be slipped through the rings, which were then pinned together, anastomosing the vessels. Ostrup and Berggren later modified this approach in 1986. They experienced no negative side effects from their coupler even three years after surgery. Couplers have grown increasingly common for executing venous anastomosis since then. The current microvascular anastomosis coupler device consists of 2 disposable rings made of high-density polyethylene, with a series of 6 to 8 (depending on the size of the coupler) stainless steel pins evenly spaced around each ring. The inner diameter of the rings range in size from 1.0 to $4.0 \mathrm{~mm}$, allowing anastomoses of vessels that are 1.0 to $4.5 \mathrm{~mm}$ in diameter [11].

Vascular couplers are considerably superior to sutures in terms of minimal preparation and intimal layer involvement. As the vessel wall is everted, it is less likely to become entrapped in the lumen [12]. When compared to micro-suturing, the anastomotic time 
is considerably reduced with couplers, but vessel wall eversion maintains patency $[5,13]$. The mean anastomosis time cited in the literature for the artery procedures was 8 minutes and for vein procedures 10 minutes.

The hemodynamic effects of using this device to anastomose tiny arteries and veins are modest, and the hemodynamic properties of the repaired vessels return over time as the body heals. The biggest issue would be the histological alterations that occur in the vessel wall since it is a rigid material that stretches the friable tiny vessels between it and the vascular clamps. The media remains viable outside the device, but it undergoes patchy necrosis inside. Intimal hyperplasia develops around and inside the device. At the component intersection, a circumferential triangular zone with loose connective and vascular channels emerges in 3 weeks. There is sometimes evidence of harm from both clamp application and the strain of approximation [14].

With the use of the device over the last few years, two main drawbacks have been pointed out. First, a ring-pin device has metallic pins on its ring that penetrate the vessel wall from the outside and permanently remain inside the vessel walls. Although the metallic pins allow very good fixation to the vessel walls, the pins can interfere with the normal restoration and the remodelling process after installation. The vessel walls are atrophied because of the continuous pressure of the blood flow against the rigidly fixed non-absorbable ring-pins complex [15]. Even though the procedure for mounting the vessels onto the device is quicker than suturing the vessel walls, metallic pins should be inserted manually by surgeons into the vessel walls. Additionally, surgeons prefer to utilise the device for venous anastomosis as the arterial wall is overly thick and rigid. The end-to-side anastomosis with the coupler is rarely performed, despite its description.

\section{Stapler Anastomosis}

In stapler anastomosis, the vessel wall ends are brought together and the stapling was done only on the outer layer. The arcuate sting shaped sharp ends of the stapler engages the vessel wall without insulting the endothelium $[16,17]$. The time required for anastomosis with a stapler is significantly less than that required for anastomosis with suturing and couplers. Despite the thicker tunica media, the stapler has been used for arterial anastomosis. After de-clamping, this displays the ability to endure pulsation. Although using staplers takes less time than using couplers, the endothelium layer is protected in both methods.

Couplers, on the other hand, are in second place to staplers in terms of cost, as they are more expensive than sutures and staplers. While the cost of couplers prevents their use, the benefit of no thrombus formation is an evident aspect that aids in the outcome. The application of couplers presents a challenge in anastomosing vessels of different calibres. In such conditions, venous anastomosis has been done in end to side fashion with good results [18].

\section{Laser-Assisted Vascular Anastomosis (LAVA)}

The main form of interaction with the tissues for LAVA is absorption. The laser light is utilised to fuse the vessel's edges together, causing adhesion by melting the collagen and coagulation of the cells. The basis of laser anastomosis is that the heat released by laser application causes denaturation of the protein composition of the tunica adventitia. As a result, a protein seal is formed at the anastomosis site. The lasers used for LAVA are the diode laser, carbon-dioxide laser, the Neodymium: Yttrium-Aluminum-Garnet laser, and the argon laser [5]. These lasers have been used in combination with different kinds of protein solutions used as solders and/or dyes in order to create an anastomosis of sufficient strength to withstand physiologic variations in blood pressure.

In the infrared spectrum, a diode laser system with a wavelength of $830 \mathrm{~nm}$ and a flexible fibre delivery system with a contact probe with a tip size of $200 \mathrm{~m}$ is used. In continuous mode, a power of $2000 \mathrm{~mW}$ is employed. According to Unno et al., prolonged light energy welds the vessel wall using methylene blue as a conducting medium [19]. The energy delivered is safe for vessel soldering and causes no tissue desiccation; However, periodic irrigation should be done after using the laser. The frequency of laser application for $3 \mathrm{e} 5 \mathrm{~s}$ results in protein coagulation and crosslinking, which leads to the formation of a seal across the anastomosis. Methylene blue dye changes colour from blue to brownish-black when the anastomosis is complete, and further application may result into charring of the vessel. The soldering agent in the surgical field is haemoglobin, which not only acts as a heatsink but also increases the surface area of the weld [20]. The additive effect of methylene blue application as a wavelength-specific chromophore allows the selective heating of the vessel, thus limiting the extent of thermal damage to the vessel.

With a rich case bank established in our institution over the last decade, we have been able to conduct research and publish extensively in the microvascular reconstruction domain [21, 30]. Future scope of the study is that a larger sample from diverse population must be assessed by implementing and monitoring the success of non-suturing methods of microvascular anastomosis in maxillofacial reconstruction over a longer period of time.

\section{Conclusion}

Suturing procedures have long been the mainstay for microvascular anastomoses, however due to technological issues and labour intensity, sutureless microvascular anastomoses have advanced significantly. Non-suturing microvascular anastomosis is a safer and more effective alternative to traditional microsuturing. Fibrin sealant reinforced microsuturing is very effective for venous anastomosis. Also, these methods are less time consuming when compared to conventional methods. This helps in reducing the flap ischemic time and increases the rate of flap survival.

\section{References}

[1]. Alan Turner MJ, Smith WP. Double venous anastomosis for the radial artery forearm flap. Improving success and minimising morbidity. J Craniomaxillofac Surg. 2009 Jul;37(5):253-7.Pubmed PMID: 19144528.

[2]. Andree C, Munder BI, Behrendt P, Hellmann S, Audretsch W, Voigt M, et al. Improved safety of autologous breast reconstruction surgery by stabilisation of microsurgical vessel anastomoses using fibrin sealant in 349 free DIEP or fascia-muscle-sparing (fms)-TRAM flaps: a two-centre study. Breast. 2008 Oct;17(5):492-8.Pubmed PMID: 18502642.

[3]. Chang TS. Principles, techniques and applications in microsurgery. World sci. 1986.

[4]. Lu Q, Liu K, Zhang W, Li T, Shi AH, Ding HF, et al. Author Correction: End-to-end vascular anastomosis using a novel magnetic compression device in rabbits: a preliminary study. Sci Rep. 2020 Oct 2;10(1):16712.Pubmed PMID: 33009495. 
[5]. Murugan S, John RR, Krihnakumar Raja VB, Mohan A, Bhanumurthy L. A Comparative Study on Microvascular Anastomosis of Vascularised Free Fibular Flap with Couplers and Suturing in Mandibular Reconstruction. J Maxillofac Oral Surg. 2016 Sep;15(3):363-366.Pubmed PMID: 27752208.

[6]. Kletter G, Matras H, Chiari H, Dinges N, Witzmann A. Comparative evaluation of conventionally sutured and clot-sutured microsurgical anastomoses. InMicrosurgery for stroke. 1977:149-153.

[7]. Cho AB, Júnior RM. Effect of fibrin adhesive application in microvascular anastomosis: A comparative experimental study. PlastReconstrSurg. 2007 Jan 1;119(1):95-103

[8]. Ritschl LM, Fichter AM, von Düring M, Mitchell DA, Wolff KD, Mücke T. Risk of Thromboembolus after Application of Different Tissue Glues during Microvascular Anastomosis. Plast Reconstr Surg. 2015 Dec;136(6):12161225.Pubmed PMID: 26267401.

[9]. Frost-Arner L, Spotnitz WD, Rodeheaver GT, Drake DB. Comparison of the thrombogenicity of internationally available fibrin sealants in an established microsurgical model. Plast Reconstr Surg. 2001 Nov;108(6):1655-60. Pubmed PMID: 11711942.

[10]. López-Monjardin H, de la Peńa-Salcedo JA. Techniques for management of size discrepancies in microvascular anastomosis. Microsurgery: Official Journal of the International Microsurgical Society and the European Federation of Societies for Microsurgery. 2000;20(4):162-6.

[11]. Zhang T, Lubek J, Salama A, Caccamese J, Coletti D, Dyalram D, et al. Venous anastomoses using microvascular coupler in free flap head and neck reconstruction. J Oral MaxillofacSurg. 2012 Apr 1;70(4):992-6.

[12]. Grewal AS, Erovic B, Strumas N, Enepekides DJ, Higgins KM. The utility of the microvascular anastomotic coupler in free tissue transfer. Can J Plast Surg. 2012 May;20(2):98-102.

[13]. Ross DA, Chow JY, Shin J, Restifo R, Joe JK, Sasaki CT, et al. Arterial coupling for microvascular free tissue transfer in head and neck reconstruction. Arch Otolaryngol Head Neck Surg. 2005 Oct 1;131(10):891-5.

[14]. Chernichenko N, Ross DA, Shin J, Chow JY, Sasaki CT, Ariyan S. Arterial coupling for microvascular free tissue transfer. Otolaryngol Head Neck Surg. 2008 May;138(5):614-8.

[15]. Ragnarsson R, Berggren A, Östrup LT. Long term evaluation of the unilink anastomotic system: a study with light and scanning electron microscopy. Scand J PlastReconstrSurg Hand Surg. 1992 Jan 1;26(2):167-71.

[16]. Mudigonda SK, Murugan S, Velavan K, Thulasiraman S, Krishna Kumar Raja VB. Non-suturing microvascular anastomosis in maxillofa- cial reconstruction- a comparative study. J Craniomaxillofac Surg. 2020 Jun;48(6):599-606.Pubmed PMID: 32402495.

[17]. Reddy C, Pennington D, Stern H. Microvascular anastomosis using the vascular closure device in free flap reconstructive surgery: A 13-year experience. J Plast Reconstr Aesthet Surg. 2012 Feb;65(2):195-200.Pubmed PMID: 21992937.

[18]. Sullivan SK, Dellacroce F, Allen R. Management of significant venous discrepancy with microvascular venous coupler. J ReconstrMicrosurg. 2003;19(06):377-80.

[19]. Unno N, Sakaguchi S, Koyano K. Microvascular anastomosis using a new diode laser system with a contact probe. Lasers Surg Med. 1989;9(2):160-8. Pubmed PMID: 2716461.

[20]. Wolf-de Jonge IC, Beek JF, Balm R. 25 years of laser assisted vascular anastomosis (LAVA): what have we learned? Eur J Vasc Endovasc Surg. 2004 May;27(5):466-76.Pubmed PMID: 15079768.

[21]. MP SK. Knowledge, Attitude and practices regarding needlestick injuries among dental students. Asian J Pharm Clin Res. 2016;9(4):312-5.

[22]. SK M. Knowledge, attitude, and practices regarding infection control among undergraduate dental students. Asian J Pharm Clin Res. 2016;9(1):220-4.

[23]. Santhosh KM, Harshini AK. Knowledge and awareness about oral cancer among undergraduate dental students. Asian J Pharm Clin Res. 2016;9(4):165-7.

[24]. Gayathri MM. Knowledge and awareness among patients about dental implants. J. Pharm. Sci. Res. 2016 May 1;8(5):351.

[25]. Vijayalakshmi B, Kumar MS. Knowledge of students about Local anaesthetics used during oral surgical procedures. J. Pharm. Sci. Res. 2015 Nov $1 ; 7(11): 1011$.

[26]. Gayathri MM. Knowledge, Awareness and Attitude among dental students about hepatitis B infection. J. Pharm. Sci. Res. 2016 Mar 1;8(3):168.

[27]. Ahamed A, Kumar MS. Knowledge, attitude and perceived confidence in handling medical emergencies among dental students. J. Pharm. Sci. Res. 2016 Jul 1;8(7):645.

[28]. Kumar S. Knowledge, attitude and practices of dental students toward dental management of patients on antiplatelet therapy. Asian J Pharm Clin Res. 2016;9(30):270-6.

[29]. Mp SK. Local hemostatic agents in the management of bleeding in oral surgery. Asian J Pharm Clin Res. 2016;9(3):35-41.

[30]. Kumar MP. Newer methods of extraction of teeth. Int J Pharm Bio Sci. 2015;6(3):679-85. 